\title{
Für die korrekte Verschreibung eines Medikamentes ist ärztliches Know-how nötig
}

\author{
Apotheker haben fundierte Kenntnisse zu Arzneimitteln, sie kennen Wirksamkeit, \\ Nebenwirkungspotential und Interaktionen. Die Verschreibung von Medikamenten \\ ist aber mehr als nur Pharmakologie auf Hochschulniveau. Wichtig sind möglichst \\ optimale Kenntnisse über die Person, die das Medikament erhält.
}

\section{Heinz Bhend}

Hausarzt und Fachlicher Leiter Institut für Praxisinformatik IPI
In der Sendung «10-vor-10» vom 6. Mai 2014 hat der Apothekerverband zur laufenden Debatte im Nationalrat betreffend Abgabe rezeptpflichtiger Medikamente folgende Stellungnahme abgegeben:

«Die Apotheker sind die durch die Hochschule qualifizierten Fachleute für die Medikamente. Wo ist das Problem?»

Meine kurze Antwort auf die kurze Frage: In der eingeschränkten Optik! Da die Apotheker offenbar kein Problem sehen, hier eine Hilfestellung:

Bei der Beschränkung des Horizontes auf die Medikamente und deren Interaktionen gibt es bezüglich Fachkompetenz des Apothekers tatsächlich kein Problem. Die Apotheken sind zudem zu nahezu 100\% digital und mit softwarebasierten Interaktionstools ausgerüstet. Die Verschreibung ist aber mehr als nur Pharmakologie auf Hochschulniveau. Die Notwendigkeit, Arzneimittel fundiert zu kennen, inklusive Wirksamkeit, Nebenwirkungspotential und Interaktionen, ist unbestritten. Etwas abstrakt formuliert kann man sagen: Die medikamentöse Behandlung beabsichtigt ja eine Interaktion zwischen Medikament und dem System «Mensch». Bei dieser Wechselwirkung sind die Medikamente die eine Seite. Genauso wichtig sind möglichst optimale Kenntnisse über die Person, die das Medikament erhält.

Um das Krankheitsbild eines Menschen nur annähernd zu erfassen ist $u$. U. das ganze Rüstzeug der Medizin nötig: Anamnese, Status und allenfalls apparative Untersuchungen. Deshalb beinhaltet die konkrete

\section{Abbildung 1}

Ein Vergleich der Medikamentenabgabe bei Arzt und Apotheker.

Doppelte Kontrolle oder „Das-4-Augenprinzip“ am Beispiel der Medikamentenabgabe

Direktabgabe ( = DMA) versus Rezeptur

Konzeptidee: Qualitätsförderung, durch „Einsatz von“ vier Augen oder zwei (Fach-) Personen um dadurch Fehler zu vermeiden.

Prozess: Kontrolle ob der richtige Patient, für die richtige Indikation, unter Berücksichtigung der Kontraindikationen, das richtige Medikament in der richtigen Dosierung (Dauer/Applikationsart) erhält.

\begin{tabular}{|c|c|c|c|c|c|c|c|}
\hline & Richtiger & Richtige & Berücksich & tigung Kontrain & dikationen & Richtiges & Richtige Dosierung \\
\hline & & & Nebendiagnosen & Laborwerte & Andere Medikamente & & \\
\hline Apotheker & $\begin{array}{l}\text { Oft wird } \\
\text { Rezept von } \\
\text { Dritten } \\
\text { eingelöst }\end{array}$ & $\begin{array}{l}\text { Diagnose ist } \\
\text { dem } \\
\text { Apotheker } \\
\text { nicht bekannt; } \\
\text { macht } \\
\text { allenfalls aus } \\
\text { Medikamenten } \\
\text { Rückschlüsse auf } \\
\text { Diagnose }\end{array}$ & $\begin{array}{l}\text { Nicht } \\
\text { bekannt }\end{array}$ & $\begin{array}{l}\text { Nicht } \\
\text { bekannt }\end{array}$ & $\begin{array}{l}\text { Bekannt, } \\
\text { Interaktions- } \\
\text { check erfolgt } \\
\text { softwarebasiert }\end{array}$ & $\begin{array}{l}\text { Keine } \\
\text { Beurteilung } \\
\text { möglich, da } \\
\text { Diagnose und } \\
\text { damit } \\
\text { Guidelines nicht } \\
\text { bekannt sind }\end{array}$ & $\begin{array}{l}\text { Standard- } \\
\text { dosierungen } \\
\text { sind keine } \\
\text { Wissenschaft, } \\
\text { da "hinterlegt". } \\
\text { Anpassungen nicht } \\
\text { möglich }\end{array}$ \\
\hline Arzt & $\begin{array}{l}\text { Kennt seine } \\
\text { Patienten, } \\
\text { bei DMA } \\
\text { erhält die } \\
\text { Person das } \\
\text { Medikament } \\
\text { unmittelbar im } \\
\text { Anschluss an die } \\
\text { Konsultation }\end{array}$ & $\begin{array}{l}\text { Diagnose und } \\
\text { damit } \\
\text { Indikation ist } \\
\text { bekannt }\end{array}$ & Bekannt & Bekannt & $\begin{array}{l}\text { Bekannt, } \\
\text { Interaktionscheck } \\
\text { erfolgt } \\
\text { softwarebasiert }\end{array}$ & $\begin{array}{l}\text { Auswahl liegt } \\
\text { alleine in der } \\
\text { Verantwort- } \\
\text { ung des } \\
\text { Arztes. Kein } \\
\text { Teamentscheid } \\
\text { möglich }\end{array}$ & $\begin{array}{l}\text { Standard- } \\
\text { dosierungen } \\
\text { sind keine } \\
\text { Wissenschaft, } \\
\text { da "hinterlegt". } \\
\text { Anpassungen an } \\
\text { Laborwerte und } \\
\text { Nebendiagnosen sind } \\
\text { möglich }\end{array}$ \\
\hline Erläuterung & & $\begin{array}{l}\text { Beinhaltet Kenntnis } \\
\text { der Diagnose }\end{array}$ & $\begin{array}{l}\text { Eine Nebendiagnose } \\
\text { kann u. U. ein } \\
\text { Hinderungsgrund sein, } \\
\text { ein Medikament } \\
\text { einzusetzen }\end{array}$ & $\begin{array}{l}\text { Laborwerte kön- } \\
\text { nen Hinderungs- } \\
\text { grund sein, ein } \\
\text { Medikament } \\
\text { einzusetzen }\end{array}$ & $\begin{array}{l}\text { Gewisse Medikamente } \\
\text { dürfen nicht } \\
\text { gleichzeitig verabreicht } \\
\text { werden (sog. } \\
\text { Interaktionen) }\end{array}$ & & \\
\hline
\end{tabular}

Korrespondenz: Dr. med. Heinz Bhend Ottnerstrasse 9 CH-4663 Aarburg info[at]dr-bhend.ch
FAZIT: Wenn das „Vier-Augen-Prinzip“ nur für Kontrolle des Rezeptes eingesetzt wird, bedeutet dies, dass eine künstlich eingebaute Schnittstelle kontrolliert wird. Mit Qualitätsförderung hat dies nichts zu tun. Diese ist gegeben, wenn eine zweite Fachperson vollumfänglich informiert ist. Um diese Zusatzinformationen (Diagnosen, Nebendiagnosen, Laborwerte) richtig interpretieren zu können, muss die Fachperson Medizinerln sein. 
